Conclusion: Therapy with TNF alpha inhibitor golimumab for 24 months in AS patients with coxitis was accompanied with statistically significant improvement of clinical scores with primary endpoint achieved (mean BASFI change -2.5 at 12 months), improvement of MRI and US findings without obvious structural progression measured with BASRI-hip score compared to baseline.

REFERENCES:

[1] Cruyssen B.V. et al. Rheumatology 2010; 49: 73-81.

[2] Boonen A. et al. J Rheumatol 2009; 36; 1249-1255.

[3] Cruyssen B.V. et al. Curr Opin Rheumatol 2013, 25: 448-454.

[4] Zhen-Guo H. et al. European Journal of Radiology 82 (2013) 1487-1493.

[5] Konsta et al. Clin Rheumatol (2013) 32:1229-1232.Braun J. et al. Ann Rheum Dis 2012; 71: 661-667.

Disclosure of Interests: Shandor Erdes Speakers bureau: Paid as a speaker during educational activities supported by pharmaceutical companies (MSD, Pfizer, AbbVie, BIOCAD), Ekaterina Agafonova Speakers bureau: Paid as a speaker during educational activities supported by pharmaceutical companies (MSD)., Daria Rumiantceva Speakers bureau: Paid as a speaker during educational activities supported by pharmaceutical companies (Novartis), Satenik Davidian: None declared, Elena Zemerova Speakers bureau: Paid as a speaker during educational activities supported by pharmaceutical companies (MSD, Pfizer), Aleksey Kulikov Speakers bureau: Paid as a speaker during educational activities supported by pharmaceutical companies (MSD, AbbVie, UCB, BIOCAD, Novartis, Sanofi), Olga Markova Speakers bureau: Paid as a speaker during educational activities supported by pharmaceutical companies (MSD, Novartis, Medac, GSK), Ekaterina Lukyanova Employee of: MSD Pharmaceutical LLC (Russia), Director of Medical Affairs., Vladimir Achikyan Employee of: MSD Pharmaceutical LLC (Russia), Therapeutic Area Lead

DOI: 10.1136/annrheumdis-2021-eular.299

\section{POS0904 FACTORS ASSOCIATED WITH SWITCHING FROM ONE ANTI-TNF AGENT TO ANOTHER ANTI-TNF, OR IL17 AGENT IN PATIENT WITH ANKYLOSING SPONDYLITIS}

M. Hwang ${ }^{1}$, M. Weisman ${ }^{2}$, L. S. Gensler ${ }^{3}$, A. Tahanan ${ }^{4}$, M. Ishimori ${ }^{2}$, T. Hunter ${ }^{5}$ R. Bolce ${ }^{5}$, J. Lisse ${ }^{5}$, M. Rahbar6, M. Shan ${ }^{5}$, J. D. Reveille ${ }^{7}{ }^{1}$ McGovern Medical School at UTHealth, Rheumatology, Houston, United States of America;

${ }^{2}$ Cedars-Sinai Medical Records Office, Rheumatology, Los Angeles, United States of America; ${ }^{3}$ University of California San Francisco Parnassus Campus, Rheumatology, San Francisco, United States of America; ${ }^{4}$ University Of Texas Professional Building, Medicine, Houston, United States of America; ${ }^{5}$ Eli Lilly, Medical Affairs, Indianapolis, United States of America; ${ }^{4}$ University Of Texas Professional Building, Medicine, Houston, United States of America; ${ }^{7}$ University of Texas McGovern Medical School, Rheumatology, Houston, United States of America

Background: A recent study examining Commercial Claims Insurance database found that many patients with ankylosing spondylitis (AS) do not remain on their initial TNF inhibitor two years after initiation, particularly women and those taking opioids.

Objectives: To examine factors associated with switching from one TNF inhibitor (i)agent to either another TNFi, IL-17i or JAKi over time (at <2years and >2 years) in a longitudinal cohort of AS patients.

Methods: Patients enrolled in the Prospective Study of Outcomes in AS (PSOAS), an observational longitudinal study of predictors of AS severity operative since 2002-2020 including over 1250 patients meeting modified New York criteria. Data collected included age, gender, ethnicity, HLA-B27 status, disease activity (BASDAI or ASDAS), erythocyte sedimentation rate (ESR), C-reactive protein (CRP), disease severity,(functional (BASFI) or radiographic (mSASSS)), comorbidities, smoking, exercise, disease duration, depression (either by self report or by the Center for Epidemiologic Studies Depression Scale (CES-D) and other medication usage (NSAIDs, including the NSAID index, nonbiologic DMARDs, opioids, anti-depressants, anxiolytics and hypnotics). Logistic regression models were built to identify clinical and sociodemographic characterstics associated with medication switching to another TNFi, IL-17i, or other biologic therapy (another TNFi, II-17i, or JAKi) within 2 years and after 2 years of initiation).

Results: Of those patients in PSOAS who had at least two years of follow-up, 496 were prescribed anti-TNF, 34 anti-IL-17 and 3 anti-JAK agents. According to the multinomial logistic regression analysis, patients who switched from their original TNFito another TNFi, IL-17i or JAKi within two years after initiating their original TNFi were more likely to be older, have higher baseline subjective disease activity (BASDAI), less radiographic severity by MSASSS, exercise $>120$ minutes/week and less likely to be currently smoking. Patients who switched after two years were less likely be depressed, had shorter disease duration, had greater subjective disease activity, were more likely to be exercising $>120 \mathrm{~min}$ utes/week, and had more comorbidities.

Conclusion: Different factors were encountered in AS patients who switched from their initial TNFi to another TNFi, IL-17i or JAKi within 2 years versus after 2 years of treatment.
Table 1. Factors Associated With Switching From One TNFi To A Second TNFi or IL-17i or JAKi Before or After Two Years Based On Multinomial Logistic Regression Model ( $\mathrm{N}=496$ Patients)

\begin{tabular}{|c|c|c|c|c|}
\hline Variable & $\begin{array}{l}\text { Switched within } \\
2 \text { years vs. not } \\
\text { switched }\end{array}$ & p-value & $\begin{array}{l}\text { Switched after } \\
2 \text { years vs. not } \\
\text { switched }\end{array}$ & p-value* \\
\hline Gender (Male vs. Female) & $\begin{array}{c}0.99 \\
(0.637,1.549)\end{array}$ & 0.98 & $\begin{array}{c}0.95 \\
(0.528,1.719)\end{array}$ & 0.87 \\
\hline HLA-B27_(+ vs. -) & $\begin{array}{c}0.99 \\
(0.639,1.523)\end{array}$ & 0.95 & $\begin{array}{c}0.66 \\
(0.365,1.192)\end{array}$ & 0.17 \\
\hline $\begin{array}{l}\text { Depression (CESD } \geq 16 \text { or } \\
\text { self-report)(Yes vs. No) }\end{array}$ & $\begin{array}{c}0.99 \\
(0.676,1.445)\end{array}$ & 0.95 & $\begin{array}{c}0.35 \\
(0.182,0.672)\end{array}$ & 0.002 \\
\hline $\begin{array}{l}\text { Disease duration at baseline } \\
\text { ( } \geq 20 \text { vs. }<20 \text { years) }\end{array}$ & $\begin{array}{c}0.72 \\
(0.485,1.062)\end{array}$ & 0.10 & $\begin{array}{c}0.27 \\
(0.146,0.491)\end{array}$ & $<0.001$ \\
\hline $\begin{array}{l}\text { Age at baseline ( } \geq 40 \text { vs. }<40) \\
\quad \text { (years) }\end{array}$ & $\begin{array}{c}2.00 \\
(1.291,3.101)\end{array}$ & 0.002 & $\begin{array}{c}1.23 \\
(0.693,2.193)\end{array}$ & 0.48 \\
\hline CRP ( $\geq 0.8$ vs. $<0.8$ ) & $\begin{array}{c}1.94 \\
(1.230,3.056)\end{array}$ & 0.004 & $\begin{array}{c}0.90 \\
(0.454,1.789)\end{array}$ & 0.77 \\
\hline BASFI ( $\geq 40$ vs. $<40$ ) & $\begin{array}{c}1.34 \\
0.852,2.118)\end{array}$ & 0.20 & $\begin{array}{c}0.87 \\
(0.450,1.688)\end{array}$ & 0.68 \\
\hline BASDAI ( $\geq 4$ vs. $<4$ ) & $\begin{array}{c}1.73 \\
(1.064,2.797)\end{array}$ & 0.03 & $\begin{array}{c}2.31 \\
(1.202,4.427)\end{array}$ & 0.01 \\
\hline NSAID index ( $\geq 50$ vs. $<50$ ) & $\begin{array}{c}1.32 \\
(0.822,2.128)\end{array}$ & 0.25 & $\begin{array}{c}0.83 \\
(0.437,1.586)\end{array}$ & 0.58 \\
\hline NSAIDs used (Yes vs. No) & $\begin{array}{c}0.84 \\
(0.534,1.309)\end{array}$ & 0.43 & $\begin{array}{c}0.85 \\
(0.479,1.510)\end{array}$ & 0.58 \\
\hline $\begin{array}{l}\text { Exercise ( } \geq 120 \text { vs. }<120) \\
\quad \text { (minutes/week) }\end{array}$ & $\begin{array}{c}1.95 \\
(1.396,2.731)\end{array}$ & $<0.001$ & $\begin{array}{c}1.66 \\
(1.057,2.613)\end{array}$ & 0.03 \\
\hline ASDAS ( $\geq 3$ vs. $<3$ ) & $\begin{array}{c}0.78 \\
(0.454,1.356)\end{array}$ & 0.39 & $\begin{array}{c}1.07 \\
(0.478,2.399)\end{array}$ & 0.87 \\
\hline $\begin{array}{l}\text { Number of comorbidities } \\
\qquad(\geq 2 \text { vs. }<2)\end{array}$ & $\begin{array}{c}1.40 \\
(0.997,1.951)\end{array}$ & 0.05 & $\begin{array}{c}1.63 \\
(1.029,2.575)\end{array}$ & 0.04 \\
\hline mSASSS ( $\geq 4$, vs. $<4$ ) & $\begin{array}{c}0.63 \\
(0.421,0.957)\end{array}$ & 0.03 & $\begin{array}{c}0.81 \\
(0.474,1.392)\end{array}$ & 0.03 \\
\hline Current smoker (Yes vs No) & $\begin{array}{c}0.69 \\
(0.385,1.225)\end{array}$ & $<0.001$ & $\begin{array}{c}0.79 \\
(0.297,2.076)\end{array}$ & 0.20 \\
\hline
\end{tabular}

*p-values calculated based on multinomial logistic regression model when switching is defined as being prescribed a second TNFi or taking IL-17i or JAKi before or after 2 years from first TNFi initiation

Disclosure of Interests: Mark Hwang Consultant of: UCB, Novartis, Michael Weisman Consultant of: Novartis, GSK, UCB, Lilly, Lianne S. Gensler Consultant of: AbbVie, GlaxoSmithKline, Eli Lilly, Novartis, Pfizer, UCB Pharma, Amirali Tahanan: None declared, Mariko Ishimori: None declared, Theresa Hunter Employee of: Eli Lilly, Rebecca Bolce Employee of: Eli Lilly, Jeffrey Lisse Employee of: Eli Lilly, Mohammad Rahbar: None declared, Minyang Shan Employee of: El Lilly, John D Reveille Consultant of: UCB, Grant/research support from: Eli Lilly DOI: 10.1136/annrheumdis-2021-eular.515

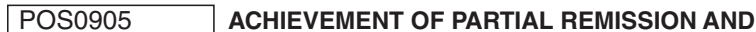 INACTIVE DISEASE IN UPADACITINIB-TREATED PATIENTS WITH ANKYLOSING SPONDYLITIS}

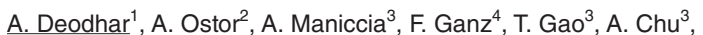

D. Poddubnyy ${ }^{5} .{ }^{1}$ Oregon Health \& Science University, Rheumatology, Portland, United States of America; ${ }^{2}$ Cabrini Hospital, Monash University \& Emeritus Research, Melbourne, Australia; ${ }^{3}$ AbbVie Inc., Rheumatology, North Chicago, United States of America; ${ }^{4}$ AbbVie, Rheumatology, Baar, Switzerland;

${ }^{5}$ Department of Gastroenterology, Infectious Diseases and Rheumatology, Charité-Universitätsmedizin, and Epidemiology Unit, German Rheumatism Research Centre, Berlin, Germany

Background: Assessment of SpondyloArthritis international Society (ASAS) response criteria and AS Disease Activity Score (ASDAS) are both commonly used, rigorous composite indices consisting of components with relevance to patients. Clinically meaningful thresholds for these measures have been defined to reflect partial remission (PR), inactive disease (ID), and low disease activity (LDA).

Objectives: To study the association of ASAS PR and ordinal ASDAS disease categories (including ASDAS ID, which is the most stringent category of this composite score) in upadacitinib (UPA)-treated patients with AS.

Methods: In the SELECT-AXIS 1 (NCT03178487) study, biologic DMARD naïve-patients (pts; $\geq 18 \mathrm{y}$ ) with active AS and intolerance/contraindication or inadequate response to $\geq 2$ NSAIDs were randomized $1: 1$ to UPA $15 \mathrm{mg}$ once daily (QD) or placebo (PBO). ${ }^{1}$ At wk 14, pts entered an open-label extension (OLE) of UPA $15 \mathrm{mg}$ QD; pts randomized to $\mathrm{PBO}$ were switched to UPA. This post hoc analysis assessed the responsiveness of individual ASAS and ASDAS core components among pts who achieved ASAS PR. The association of ASAS PR with achievement of ASDAS ID (ASDAS <1.3), ASDAS LDA (ASDAS <2.1 but $\geq 1.3$ ) or ASDAS high disease activity (HDA)/very HDA (VHDA) (ASDAS $\geq 2.1$ for HDAVHDA) was also assessed by measures including Youden index, distance to perfect point, and sensitivity/specificity 